\title{
Opposite Effects of Metformin against Non-Alcoholic Fatty Liver Disease, Depend on Steatosis Grade
}

\author{
Takayama F1, Fujihara $\mathrm{Y}^{* 1}$, Tsuno W1, Kamatani $\mathrm{H}^{1}$, Kabuto $\mathrm{H}^{2}$ and \\ Mankura $\mathbf{M}^{3}$ \\ ${ }^{1}$ Department of Frontier Health Sciences, Graduate School of Medicine, Dentistry and \\ Pharmaceutical Sciences, Okayama University, Japan \\ ${ }^{2}$ Kagawa Prefectural College of Health Sciences, Japan \\ ${ }^{3}$ Kurashiki Sakuyo University, Japan
} Mini Review

Volume 4 Issue 2

Received Date: May 24, 2019

Published Date: May 29, 2019

DOI: $10.23880 /$ act-16000157

*Corresponding author: Yuri Fujihara, Department of Frontier Health Sciences, Graduate School of Medicine, Dentistry and Pharmaceutical Sciences, Okayama University, Okayama 700-8530, Japan, Tel: 81-86-251-7973; Email: takayamf@okayama-u.ac.jp

\begin{abstract}
Currently, as there are no standardized treatment regimen for non-alcoholic fatty liver disease (NAFLD) and the assumption of insulin resistance involved in NAFLD, so, the effectiveness of medicines of type 2 diabetes mellitus including metformin is expected. Despite of the precise mechanism remaining controversial, metformin has been reported to activate AMP-activated protein kinase (AMPK) which is a master regulator of energy homeostasis, and is activated in response to an energy shortage imposed by physical activity and caloric restriction. In our study to investigate the metformin effectiveness on NAFLD, the opposite effects were found. Specifically, there has been shown that metformin well ameliorated mild steatosis, but aggravated high grade of steatosis to hepatitis and fibrosis (NASH), which were seemed closely related to liver mitochondrial dysfunction. The molecular mechanism of metformin to activate AMPK was demonstrated by the consequence to inhibit the complex I of mitochondrial respiratory chain, which is interpreted as a poison to mitochondrial energy metabolism, especially, when a certain degree damage exists in mitochondria. So, we here put the results and state opinion for expansion of metformin indications safely and efficiently.
\end{abstract}

Keywords: AMPK; Complex I; Insulin Resistance; Metformin; Mitochondria; Non-Alcoholic Fatty Liver Disease; NonAlcoholic Steatohepatitis

Abbreviations: NAFLD: Non-Alcoholic Fatty Liver Disease; AMPK: AMP-activated protein kinase; NASH:
Non-Alcoholic Steato-Hepatitis; T2DM: Type 2 Diabetes Mellitus; CL: Chemi-Luminescence. 


\section{Introduction}

The incidence rate of non-alcoholic fatty liver disease (NAFLD) is reported to be much higher with the patients of diabetes mellitus, hyperlipidemia and obese patients [1]. NAFLD includes a wide spectrum of conditions that range from a simple steatosis to nonalcoholic steatohepatitis (NASH) which may easily progress to cirrhosis, its complications and cancer. A growing body of evidence proposes that ectopic fat accumulations are linked to insulin resistance, metabolic disturbances and are accompanied by a state of chronic, low-grade inflammation, systemic oxidative stress and that increases risk for metabolic syndrome-associated diseases. The pathogenesis of NAFLD is not well understood, however, insulin resistance and metabolic syndrome have been associated with NAFLD [2]. Currently, there is no standardized treatment regimen for NAFLD, so, the intervention using medicines of type 2 diabetes mellitus (T2DM) including metformin and insulin sensitizer have been performed, as NAFLD involving in insulin resistance $[3,4]$.

\section{Efficacy of Metformin against NAFLD including NASH}

Efficacies of metformin against NAFLD were demonstrated in animal experiment researches and clinical experiment researches [4]. In our study using metformin and pioglitazone an insulin sensitizer against NAFLD, surprising, it was exhibited the opposite effects of metformin against fatty liver, depending on the degree of steatosis, as it well ameliorates mild/uncomplicated steatosis (Figure 1, B1), but aggravated high grade of steatosis (Figure 1, C1) to hepatitis (NASH) and hepatic fibrosis (Figure 1, C2). The NAFLD rats with mild/uncomplicated steatosis and those with high grade of steatosis were prepared by feeding with cholinedeficient, high-fat and sugar-enriched (CDHF) diets [5] for 20 days and for 30 days, respectively, followed by oral metformin $(100 \mathrm{mg} / \mathrm{kg})$ administration. By the detection of the chemiluminescence (CL) from the luminol oxidized by reactive oxygen species (ROS) [5], there exhibited the increase of ROS derivation from liver mitochondria energy metabolism in NAFLD rats by feeding with CDHF diets for 20 days and for 30 days, compared to the control rats fed with standard diets for rodents (Figure 2), which reflects mitochondrial dysfunction. Obviously, as longer period fed with CDHF, the more likely is liver mitochondrial ROS derivation and the grade of steatosis. Metformin relieved the liver mitochondrial ROS derivation of rats with mild/uncomplicated steatosis, and exacerbated that with high grade of steatosis. Incidentally, our study also demonstrated that pioglitazone relived NAFLD, regardless of the steatosis grade and the degree of ROS derivation and that metformin worked more effectively against mild/uncomplicated steatosis (data not shown).

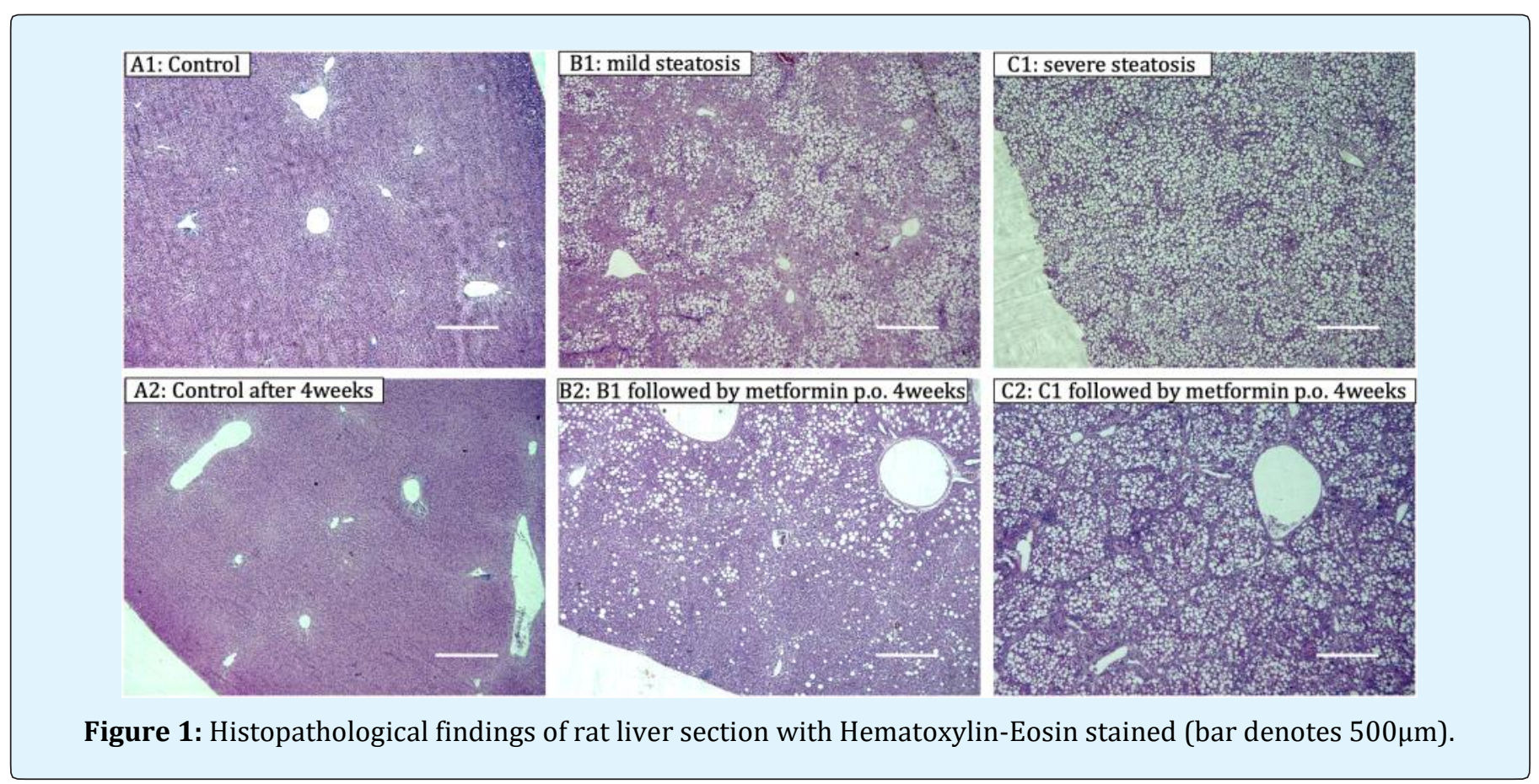




\section{Advances in Clinical Toxicology}
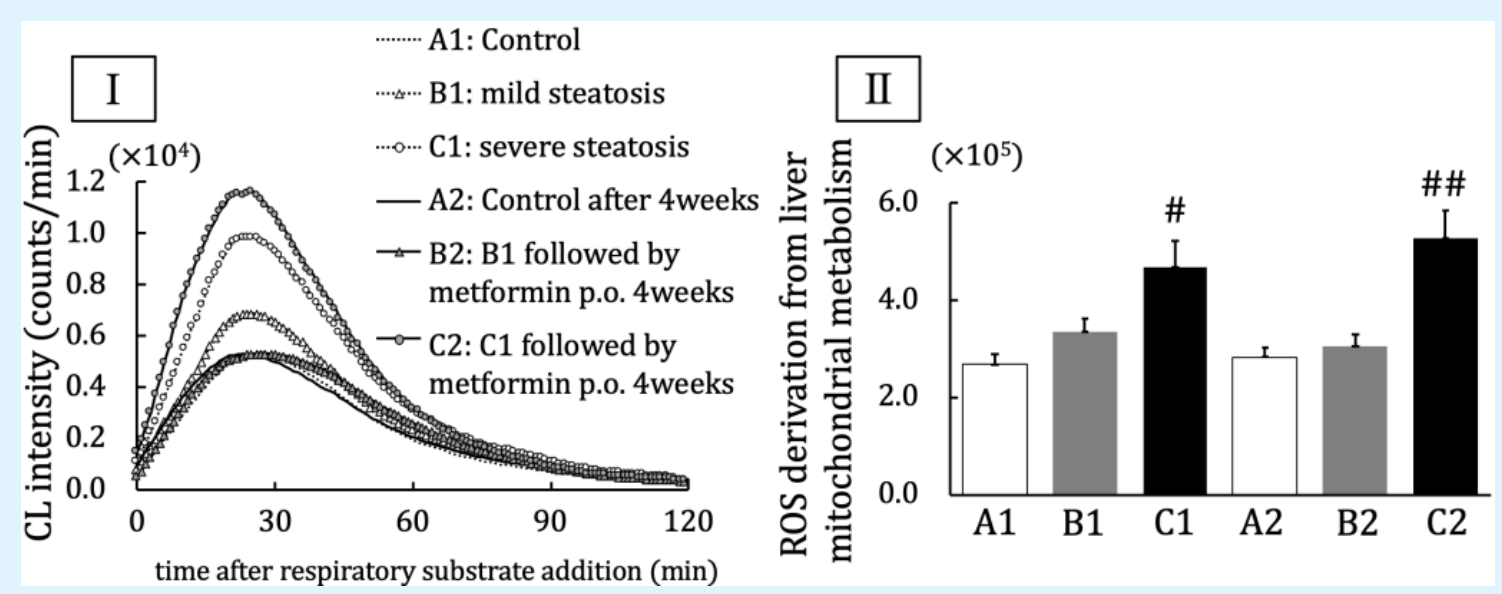

Figure 2: Reactive oxygen species (ROS) derived from mitochondrial energy metabolism.

I. The typical chemiluminescence (CL) curve detected with each group. ROS derivation was detected by luminol CL elicited by the reaction with ROS.

II. The ROS derivation amounts was calculated from the accumulated CL intensity detected for $120 \mathrm{~min}$.

$\# p<0.05, \# \# p<0.01$ vs Control group. (ANOVA with Tukey-Kramer multiple comparison test).

\section{Discussion}

Metformin has become the first medication prescribed for T2DM to lower blood glucose in world-wide, by the FDA approval 1994 for T2DM and by the report 1998 of United Kingdom Prospective Diabates Study [6]. Despite almost 60 years of clinical use, the precise mechanism of metformin remains controversial [7]. Beyond improving glycaemic control, there are shown the efficacy against NAFLD, anti-canser effects and are expected to exert prolongevity effects and increase lifespan [8]. However, against the metformin effectiveness, there is the objection [9]. Nonetheless, we expected and investigated the metformin efficacy against NAFLD, by assuming metformin as AMPK activator like exercise, and by already confirming our NASH animal model [5] accompanied with insulin resistance. The opposite effects of metformin, depending on NAFLD progression detected by our result. Still, there is the meta-analysis study of randomized controlled trials of pharmacologic agents in clinical NASH patients that reported no significant improvement for liver histologic scores for steatosis, ballooning, and fibrosis and worsening lobular inflammation significantly by metformin [4]. The molecular mechanism of metformin remains unclear, however, it has been suggested to activate AMPK, at least partially, by the consequence to inhibit the complex I of mitochondrial respiratory chain, the powerhouses [10]. The complex I inhibition is interpreted as a poison to mitochondrial energy metabolism, especially, when a certain degree damage exists in mitochondria. The worsened effect of metformin for NAFLD is guessed to induce by the mitochondrial complex I inhibition which will further attack the mitochondrial dysfunction resulting in degradation of hepatic functional reserve and the hepatocyte death.

\section{Conclusion}

This article warns the risk of metformin to exacerbate metabolic syndrome and associated diseases such as NAFLD accompanied with severe mitochondrial dysfunction, in spite of its effectiveness for allied diseases without or with the less mitochondrial dysfunction. The mitochondrial complex I inhibition is interpreted as a poison to mitochondrial energy metabolism.

\section{Funding}

This work was supported by JSPS KAKENHI Grant Numbers 25350886.

\section{References}

1. Alam S, Mustafa G, Alam M, Ahmad N (2016) Insulin Resistance in Development and Progression of Nonalcoholic Fatty Liver Disease. World J Gastrointest Pathophysiol 7(2): 211-217. 


\section{Advances in Clinical Toxicology}

2. Tangvarasittichai S (2018) Hypertriglyceridemia and Non-Alcoholic Fatty Liver Disease in Metabolic Syndrome and Type 2 Diabetes. Diabetes Obes Int J 3(4): 000188.

3. Omer Z, Cetinkalp S, Akyildiz M, Yilmaz F, Batur Y, et al. (2010) Efficacy of Insulin-Sensitizing Agents in Nonalcoholic Fatty Liver Disease. Eur J Gastroenterol Hepatol 22(1): 18-23.

4. Said A, Akhter A (2017) Meta-Analysis of Randomized Controlled Trials of Pharmacologic Agents in NASH. Ann Hepatol 16(4): 538-547.

5. Pak W, Takayama F, Mine M, Nakamoto K, Kodo Y, et al. (2012) Anti-Oxidative and Anti-Inflammatory Effects of Spirulina on Rat Model of Non-Alcoholic Steatohepatitis. J Clin Biochem Nutr 51(3): 227-234.

6. King P, Peacock I, Donnelly R (1998) The UK Prospective Diabetes Study (UKPDS): Clinical and
Therapeutic Implications for Type 2 Diabetes. Br J Clin Pharmacol 48(5): 643-648.

7. Kinaan M, Ding H, Triggle CR (2015) Metformin: An Old Drug for the Treatment of Diabetes but a New Drug. Med Princ Pract 24(5): 401-415.

8. Zhou J, Massey S, Story D, Li L (2018) Metformin: An Old Drug with New Applications. Int J Mol Sci 19(10): 2863.

9. El-Eshmawy MM (2018) Metformin: Is It A Truly Magic Drug? Diabetes Obes Int J 3(2): 000177.

10. El-Mir MY, Nogueira V, Fontaine E, Avéret N, Rigoulet $M$, et al. (2000) Dimethylbiguanide inhibits cell respiration via an indirect effect targeted on the respiratory chain complex I. J Biol Chem 275(1): 223228. 